\title{
Identification of the angiogenesis related genes for predicting prognosis of patients with gastric cancer
}

\author{
Sheng Zheng ${ }^{1,2+}$, Zizhen Zhang ${ }^{1,2+}$, Ning Ding ${ }^{1,2}$, Jiawei Sun ${ }^{1,2}$, Yifeng Lin ${ }^{1,2}$, Jingyu Chen ${ }^{1,2}$, Jing Zhong ${ }^{1,2}$, \\ Liming Shao ${ }^{1}$, Zhenghua Lin ${ }^{1,2}$ and Meng Xue , $^{1,2^{*}}$
}

\begin{abstract}
Introduction: Angiogenesis is a key factor in promoting tumor growth, invasion and metastasis. In this study we aimed to investigate the prognostic value of angiogenesis-related genes (ARGs) in gastric cancer (GC).

Methods: mRNA sequencing data with clinical information of GC were downloaded from The Cancer Genome Atlas (TCGA) and the Gene Expression Omnibus (GEO) databases. The differentially expressed ARGs between normal and tumor tissues were analyzed by limma package, and then prognosis-associated genes were screened using Cox regression analysis. Nine angiogenesis genes were identified as crucially related to the overall survival (OS) of patients through least absolute shrinkage and selection operator (LASSO) regression. The prognostic model and corresponding nomograms were establish based on 9 ARGs and verified in in both TCGA and GEO GC cohorts respectively.

Results: Eighty-five differentially expressed ARGs and their enriched pathways were confirmed. Significant enrichment analysis revealed that ARGs-related signaling pathway genes were highly related to tumor angiogenesis development. Kaplan-Meier analysis revealed that patients in the high-risk group had worse OS rates compared with the low-risk group in training cohort and validation cohort. In addition, RS had a good prognostic effect on GC patients with different clinical features, especially those with advanced GC. Besides, the calibration curves verified fine concordance between the nomogram prediction model and actual observation.
\end{abstract}

Conclusions: We developed a nine gene signature related to the angiogenesis that can predict overall survival for GC. It's assumed to be a valuable prognosis model with high efficiency, providing new perspectives in targeted therapy.

Keywords: Gastric cancer, Angiogenesis, Prognostic, Gene

\section{Introduction}

Gastric cancer (GC) is a common gastrointestinal malignancy which is the fifth most frequently diagnosed cancer $(1,000,000$ case/year) and the third leading cause of

\footnotetext{
*Correspondence: xuemeng@zju.edu.cn

†Sheng Zheng and Zizhen Zhang contributed equally to this work

${ }^{1}$ Department of Gastroenterology, The Second Affiliated Hospital

of Zhejiang University School of Medicine, 88 Jiefang Road,

Hangzhou 310009, Zhejiang, China

Full list of author information is available at the end of the article
}

cancer-related deaths $(783,000$ case/year) toll worldwide [1]. Even if diagnostic and therapeutic strategies have been improved over the past 20 years, the outcome is still poor with overall 5-year survival rate less than 40\% [2-4]. The discovery and application of molecular biomarkers have improved the prognosis evaluation and recognition of potential high-risk GC patients [5]. Therefore, it is of great significance to clarify the interactions of key molecules during the occurrence and development of GC, 
which will help to prevent GC and find new therapeutic targets.

Angiogenesis is the formation of new blood vessels from pre-existing ones through a process called germination [6]. It is mainly involved in embryonic development and wound healing under physiological conditions [7]. Without vascular supply, the tumors cannot grow over 1-2 $\mathrm{mm}$, so pathological angiogenesis is one of the hallmarks of the tumor $[8,9]$. In order to support the high proliferation rate and high metabolic rate of cancer cells, it's required to develop new vascular networks rapidly, which is driven by angiogenic factors [10]. These neonatal blood vessels not only provide the necessary oxygen and nutrients for maintaining the rapid growth and proliferation of tumor cells, but also provide the possibility for tumor cells to enter the circulatory system and metastasize to the distance [11].

The expression pattern of angiogenesis factors was closely related to the prognosis of GC patients and has been studied as a therapeutic target [12]. For example, Ramucirumab (an VEGFR-2 antibody) can significantly prolonged overall survival (OS) of GC patients [13]. It has been approved by the Food and Drug Administration (FDA) as a second-line treatment for advanced GC in 2014. However, only few angiogenic factors have been determined to be associated with the prognosis of GC patients. Besides, most studies focused on the effect of a single gene on the prognosis, such as VEGF [14]. It will be more effective to identify the prognosis of GC by combining the expression levels of various angiogenesis factors.

In the present study, the clinical data of $375 \mathrm{GC}$ patients were downloaded from The Cancer Genome Atlas (TCGA), and the correlation between the expressions of angiogenesis-related genes (ARGs) were analyzed. Besides, risk score (RS) was calculated as an independent index for overall survival (OS) prognosis based on ARGs. These findings reveal some key AGRs in gastric cancer and have a certain guiding significance for the follow-up study of tumor angiogenesis. In addition, these results can also provide an effective risk score formula for predicting the prognosis and guiding the management of GC patients.

\section{Materials and methods}

\section{Selection of angiogenesis-related genes}

AGRs were retrieved from the GeneCards website (https://www.genecards.org/) with the term "angiogenesis". Relevance scores was used to indicate the intensity of the correlation between genes and angiogenic activity, ranging from 0 to 100 . A high score represents a strong correlation. The ARGs with correlation scores $>5$ were screened for follow-up study and analysis.

\section{Acquisition of gastric cancer datasets}

The original RNA-sequencing (RNA-seq) datasets and clinical characteristics of the TCGA gastric cancer cohort were downloaded from the TCGA website (https://portal.gdc.cancer.gov/). GSE84437, including 433 GC samples dataset, was obtained from the Gene Expression Omnibus (GEO, https://www.ncbi.nlm.nih.gov/geo/) for the validation study.

\section{Differentially expressed ARGs and enrichment analysis}

The differentially expressed ARGs in the mRNA expression data of GC cohort were identified by the limma package in $\mathrm{R}$ software $(\mathrm{FDR}<0.05,|\log \mathrm{FC}|>1)$. Visualization (with volcano plots and heatmaps) was performed using the ggrepel, ggplot, and pheatmap packages in $\mathrm{R}$ software. The functional annotations of these ARGs by Gene Ontology (GO), including biological process, cellular component, and molecular function, were analyzed and visualized with the goplot package. Similarly, the KEGG pathway enrichment analysis was implemented from the KEGG pathway database (www.kegg.jp/kegg/ kegg1.html).

\section{Protein-protein interaction network construction and module screening}

The protein-protein interactions (PPIs) of all differentially expressed ARGs were identified using the STRING database (http://www.string-db.org/). The further constructed and visualized of the PPI network was used by Cytoscape 3.7.0 software. Subsequently, the important modules and genes were screened from the PPI network with scores $>5$ and node counts $>5$ by the MCODE (Molecular Complex Detection) plug-in.

\section{Establishing an individualized prognostic index according to ARGs}

Combining mRNA expression levels of ARGs and clinical data, the differentially expressed ARGs with significant prognostic value were screened by univariate Cox regression analysis. Identified overall survival (OS) related genes were used to develop prognostic multiplegene signatures. Least absolute shrinkage and selection operator (LASSO) Cox regression method was adopted to construct multivariable models with ARGs using the "glmnet" package for $\mathrm{R}$ software $[15,16]$. In LASSO regression, only the genes with non-zero coefficient are selected to further calculate the risk score [17]. The best model is determined by maximizing the performance and using the least number of genes.

Subsequently, based on the linear combination of the expression level and the weighted regression coefficient 
obtained by LASSO Cox regression analysis, the prognostic risk score formula was established. Risk score $(\mathrm{RS})=$ expression of gene $1 \times \beta 1+$ expression of gene $2 \times \beta 2+\cdots+$ expression of gene $\mathrm{n} \times \beta \mathrm{n}$. The median RS was chosen as a cutoff value to separately dichotomize TCGASTAD cohorts into high-risk and low-risk subgroups. Univariate and multivariate Cox regression analysis were used to evaluate the prognostic value of these genes in patients with GC. The survival curve was drawn by Kaplan-Meier (KM) method, and the difference of survival rate between high risk group and low risk group was evaluated by logrank test. Receiver operating characteristic (ROC) analyses were performed in R "survival ROC". Moreover, the clinical significance of these identified genes was evaluated. Then, these findings were tested in another GC cohort in GEO datasets through survival analysis and ROC curve analysis. Furthermore, the nomogram with calibration plots was built using rms $\mathrm{R}$ package to forecast the concordance between actual and predicted survival.

\section{Statistical analysis}

All statistical analyses and plots were implemented through $\mathrm{R}$ software (version 3.6.0). The correlation between risk score and clinical characteristics was tested by $\mathrm{X}^{2}$ test. The Kaplan-Meier curve was drawn and the log-rank test was used to test the significant difference of OS among the groups. Univariate and multivariate Cox proportional hazard regression analyses were also used to evaluate the relationship between risk scores and OS. ROC analysis was used to detect the sensitivity and specificity of gene signature risk score in predicting survival. The area under ROC curve (AUC) can be used as an index of prognostic accuracy. In all analyses, $P$ value $<0.05$ was set to be statistically significant.

\section{Results}

Identification of differentially expressed ARGs

RNA-seq and clinical follow-up data were downloaded from TCGA-STAD dataset, including 375 gastric cancer samples and 32 normal samples. The demographic and clinical features of these patients were listed in Additional file 1: Table I. A total of 338 angiogenesis-related genes with relevance scores $>5$ were acquired from GeneCards database. Then, we extracted and compared the expression level of 338 ARGs in RNA-seq data of normal samples and GC samples. The results indicated that there were 61 genes significantly upregulated and 24 genes significantly downregulated in GC (Fig. 1a, b).

\section{Biological functions and significant pathway analysis involved in the expression of ARGs}

The biological functions and important pathways of 85 differentially expressed ARGs were analyzed. GO enrichment analysis showed that differential ARGs played an important role in angiogenesis and vasculature development in GC (Fig. 2a, b). The results of KEGG pathway enrichment analysis showed that the differentially expressed ARGs were mainly involved in the pathways related to tumor angiogenesis, including PI3K-Akt, MAPK and Rap1 pathways (Fig. 2c, d).

\section{Construction of PPI network and selection of key modules}

To further understand the potential molecular functions of differently expressed ARGs in STAD, we constructed the PPI network using Cytoscape software which incorporated 86 nodes and 752 edges based on the data from STRING database (Fig. 3a). Then, the co-expression network was further analyzed to detect potential critical

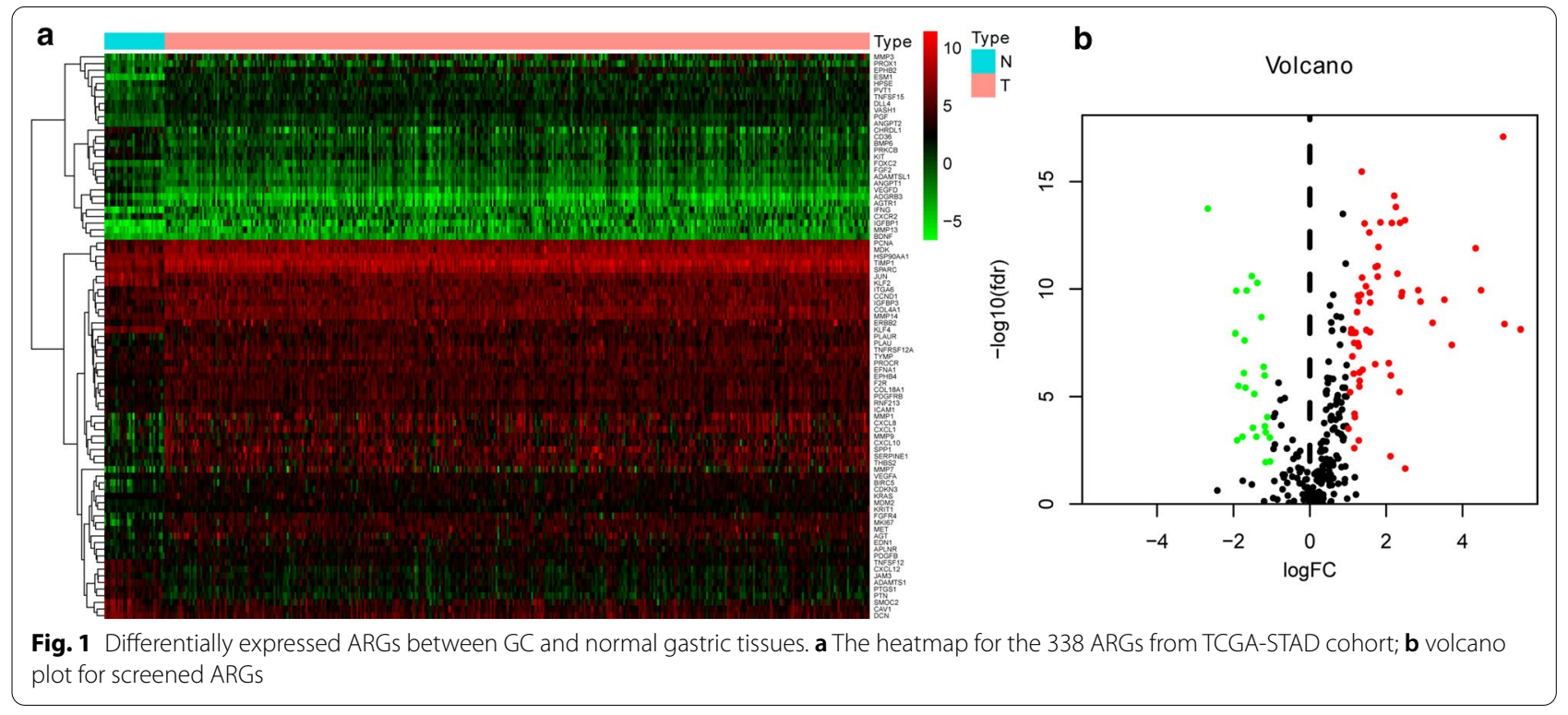




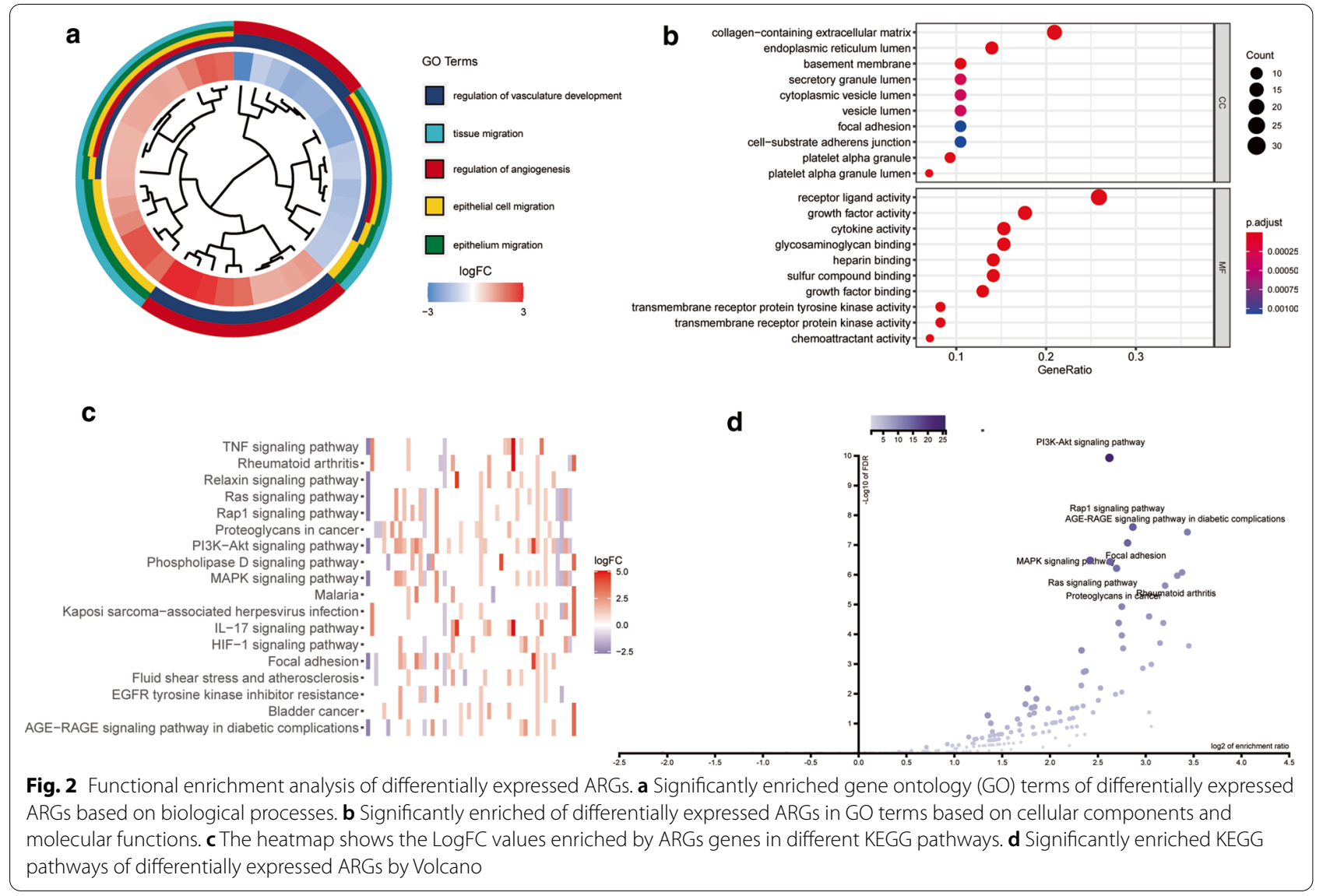

modules via the MODE tool in Cytoscape. The top two significant modules, module 1 including 15 nodes and 83 edges (Fig. 3b), and module 2 consisting of 19 nodes and 97 edges were identified (Fig. 3c). The GO and pathway analyses showed that the genes from module 1 were mainly enriched in positive regulation of chemotaxis, epithelial cell migration, and vasculature development, whereas the genes in module 2 were significantly enriched in collagen catabolic process, extracellular matrix organization, leukocyte migration, and mechanical stimulus.

\section{Construction of an angiogenesis-related prognostic model} Univariate Cox regression analysis was used to analyze the correlation between the transcriptional expression of 85 differentially expressed ARGs and clinical data. It was found that 18 genes had significant associations $(P<0.05)$ with the prognosis of GC patients (Additional file 1: Table II). The LASSO COX regression analysis of 18 significant genes was implemented, and 9 genes (AGT, ANGPT1, SERPINE1, ANGPT2, PVT1, PROCR, KIT, PLAUR and CAV1) were screened out which could be the independent prognostic predictor in GC (Fig. 4a, b). The contribution rate of the 9 genes to the risk scoring model was weighted by the absolute value of the coefficient. According to the result of LASSO Cox regression analysis, the formula of the RS is as follows: RS $=0.035291 *$ expression value of CAV1 $+0.084699 *$ expression value of PLAUR $+0.125582 *$ expression value of $\mathrm{KIT}+0.030171 *$ expressionvalueofAGT $+0.055867 *$ expression value of ANGPT $1+0.119573 *$ expression value of SERPINE1 $+0.085701 *$ expression value of ANGPT $2+0.094991 *$ expression value of PROCR $-0.181397 *$ expression value of PVT1.

In addition, univariate COX analysis and multivariate COX analysis were performed on the STAD-TCGA cohorts to further verify the reliability of RS in predicting the prognosis of patients with GC (Fig. 4c, d). The results showed that whether univariate COX regression analysis (HR 3.599, $95 \%$ CI 2.180-5.943, $P<0.001$ ) or multivariate COX regression analysis (HR 3.566, 95\% CI 2.118-6.004, $P<0.001$ ), after adjusting other clinical features such as age, gender, tumor histological grade (grade) and TNM stage $(T, N, M)$, RS was still an independent prognostic factor for GC patients. 

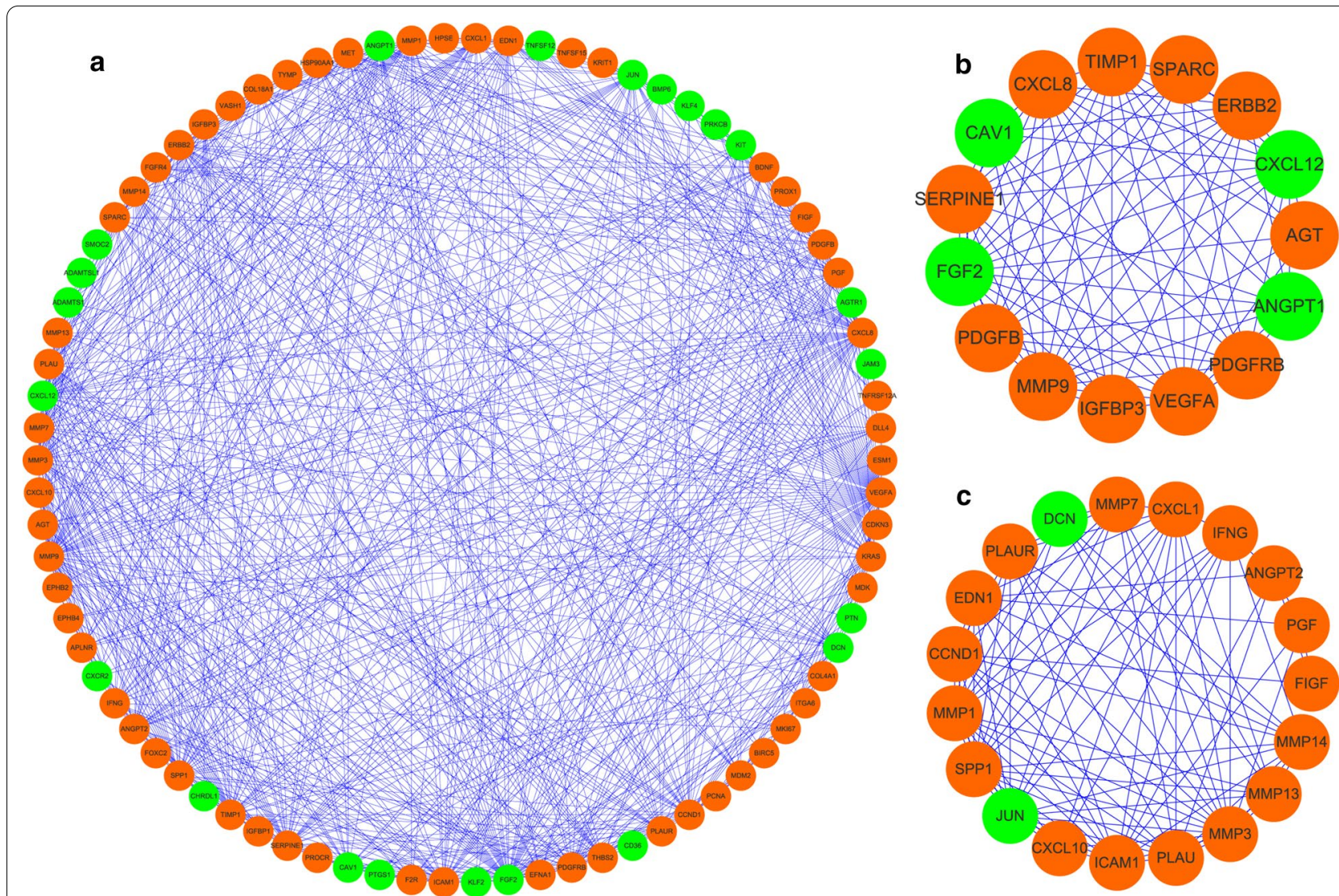

Fig. 3 PPI network and module analysis. a The PPI network of all the differentially expressed ARGs visualized by Cytoscape. b Critical module 1 in PPI network. c Critical module 2 in PPI network

\section{Validation of the angiogenesis-related prognostic model} This angiogenesis-based prognostic signature could work as a predictive tool to evaluate the prognosis of patients with gastric cancer. In the training group from TCGA-STAD cohort, each patient will get a RS based on the expression of 9 ARGs. Taking the median RS in all patients as the cut-off value, the whole group was divided into high-risk group and low-risk group. There were significant differences in RS distribution, vital status of patients and heatmap of the 9 ARGs expression profiles between high risk group and low risk group (Fig. 5a). The OS in the low-risk group was significantly better than that in the high-risk group $(P<0.001)$ (Fig. $5 \mathrm{c})$. ROC curve (AUC) analysis showed that RS has a considerable diagnostic and prognostic values on patients with GC $(A U C=0.795)$ (Fig. 5d). Then, we employed a validation group in GSE84437 cohort from GEO datasets with 433 GC cases and used OS to verify the effectiveness of the angiogenesis genes prognostic model. As expected, the validation group patients in the high-risk group distinguished by the prognostic model had a worse OS than those in the low-risk group (Fig. 5b, e). Moreover, the ROC curve shows the same results (Fig. 5f).

Then the patients in the training group were stratified according to the clinical characteristics, and the correlation between RS and OS in patients with GC was analyzed. The results showed that under the stratification of different clinical characteristics, RS had a good prognostic effect on patients with GC, especially in patients with advanced GC (Fig. 6a-f). In addition, we found that the expression of VEGFA was significantly up-regulated in tumor tissues (Additional file 1: Fig. 1a). However, in TCGA and GEO databases, the expression of VEGFA had no significant association with the prognosis of GC patients (Additional file 1: Fig. $1 \mathrm{~b}$ and $\mathrm{c}$ ).

\section{Construction of a nomogram}

According to Cox regression combined with the significant clinical parameters, the parameters contains age, stage and risk score were selected to construct nomogram (Fig. 7a). Each patient will get a score according to the prognostic 


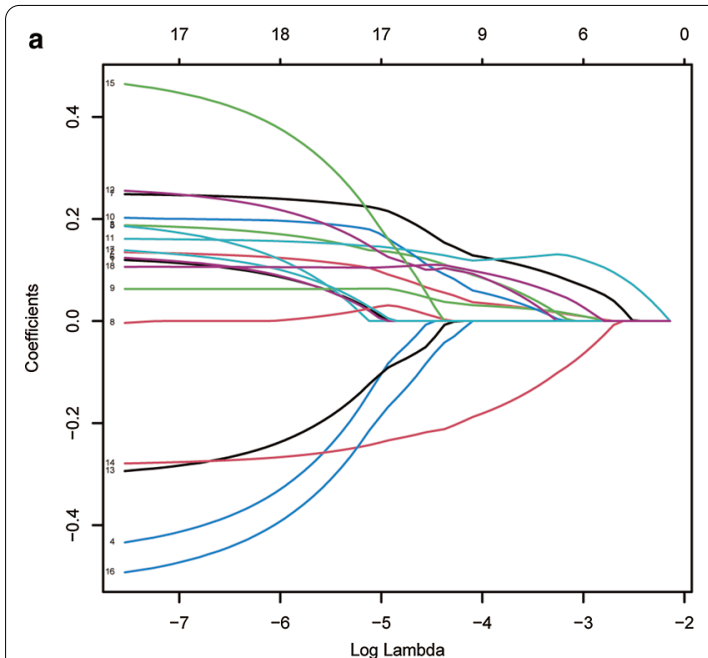

C

$\begin{array}{lrr} & \text { pvalue } & \text { Hazard ratio } \\ \text { age } & 0.006 & 1.027(1.008-1.046) \\ \text { gender } & 0.062 & 1.484(0.980-2.247) \\ \text { grade } & 0.095 & 1.368(0.947-1.977) \\ \mathrm{T} & 0.032 & 1.298(1.023-1.645) \\ \mathrm{M} & 0.025 & 2.048(1.096-3.827) \\ \mathrm{N} & 0.006 & 1.267(1.069-1.502) \\ \text { riskScore } & <0.001 & 3.599(2.180-5.943)\end{array}$

univariate Cox regression

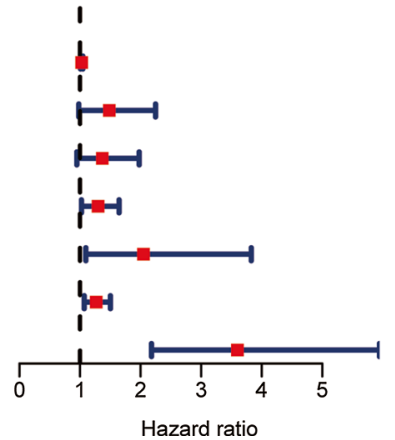

b

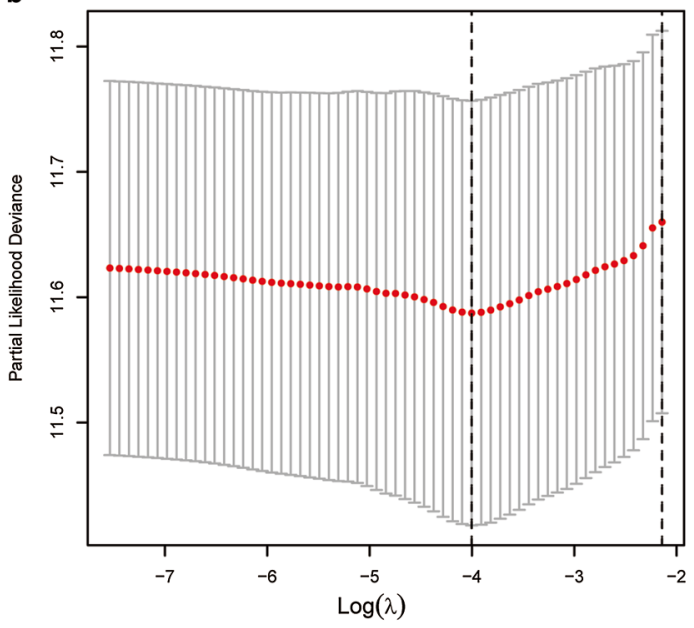

d

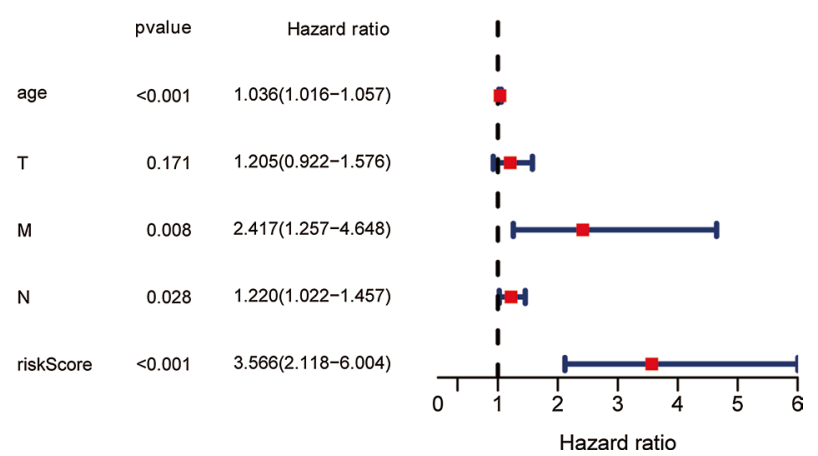

Fig. 4 Establishment of ARGs prognostic model related to the prognosis of GC by lasso regression model. a LASSO coefficient profiles of the 18 ARGs. b A coefficient profile plot was generated against the log (lambda) sequence. c Univariate COX regression analysis for RS of GC patients in TCGA database. d Multivariate Cox regression analysis for RS of GC cancer patients in TCGA datasets

parameters, and the higher the total score indicates a worse outcome. Moreover, the ROC curves of 1-, 3- and 5-year OS indicated that our model has good predictive ability (Fig. 7b). Calibration plots had demonstrated a great consistency between the predicted and observed outcomes (Fig. 7c).

\section{Discussion}

Gastric cancer is considered to be one of the most malignant tumors in the world due to high recurrence rate and low survival rate [18]. For clinicians, it is still challenging to predict the prognosis and risk stratification of patients with gastric cancer. Due to the limitations of TNM staging system and other scoring systems, there is an urgent need for new molecular biomarkers to predict the survival of patients with GC [19]. In the current study, we constructed and validated a gastric cancer risk model based on ARGs. As far as we know, this is the first study to explore prognostic biomarkers in patients with $\mathrm{GC}$ using angiogenesis-related gene scoring model.

Angiogenesis is a physiological process during tissue repair and regeneration, such as reproduction, embryonic development and wound healing [20]. Under normal quiescent, endothelial cells can sense angiogenic signals and participate in angiogenesis by maintaining a high degree of plasticity under controlled conditions. However, in many disease states, such as cancer, rheumatoid arthritis and atherosclerosis, uncontrolled angiogenesis will further promote the development of the disease and become a hallmark of these disease states [21]. The growth and metastasis of gastric cancer also depend on angiogenesis. After being stimulated by hypoxia and energy deficiency, tumor cells and tumor microenvironment will release a large number of angiogenic factors to trigger angiogenesis. Then 

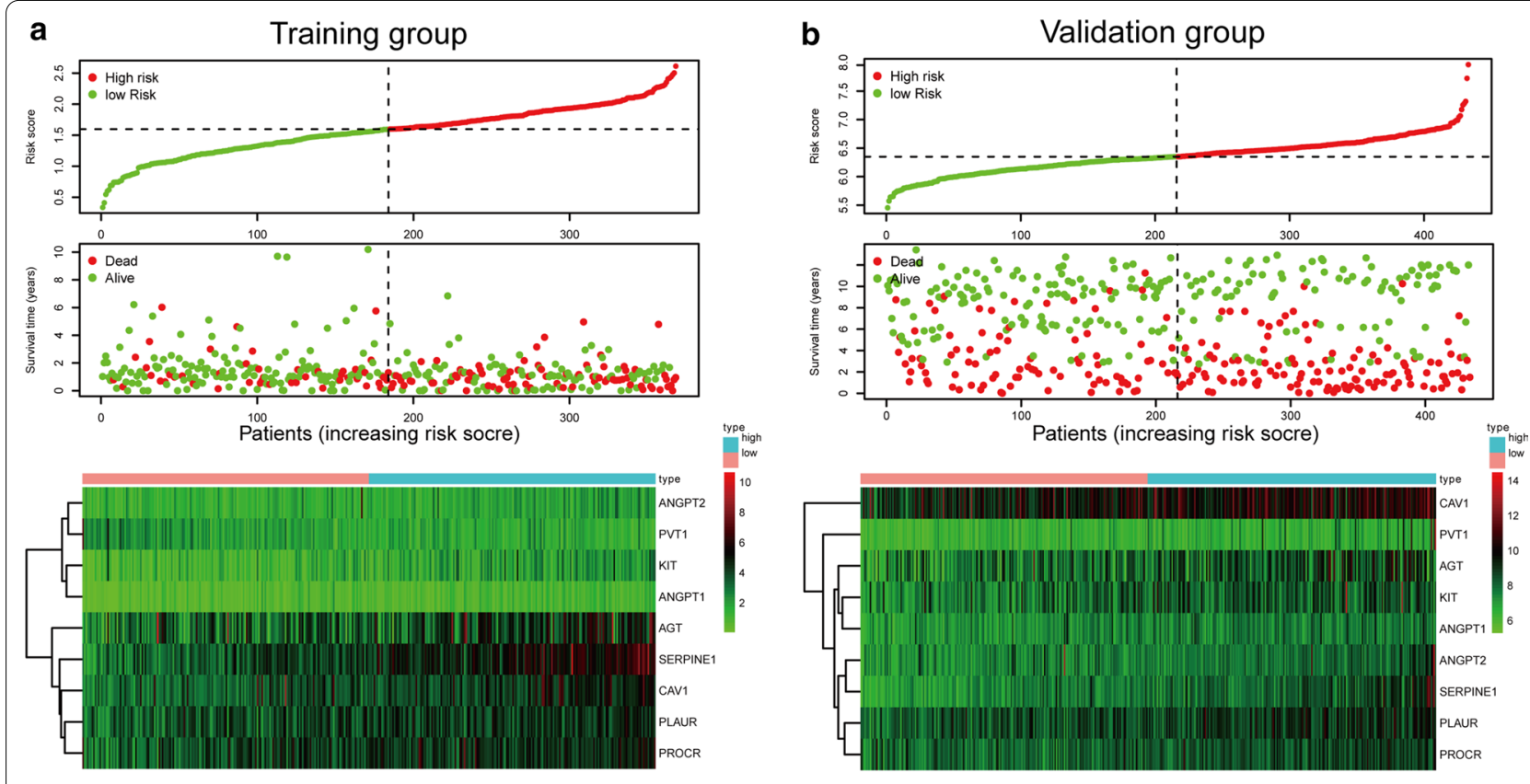

C

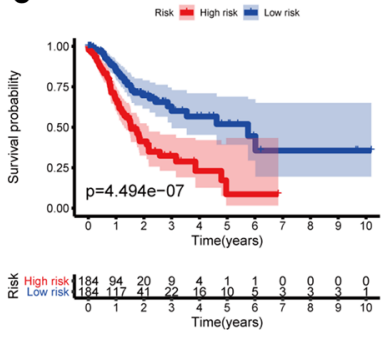

d

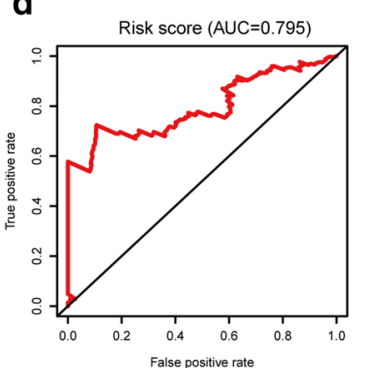

e

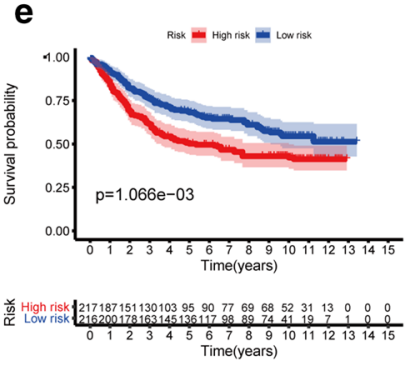

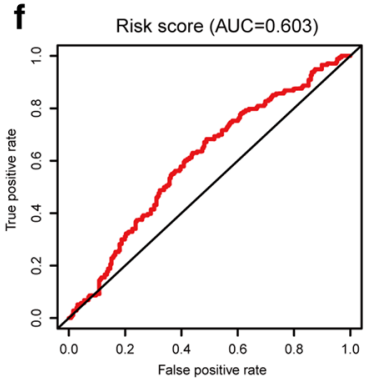

Fig. 5 Development of RS based on the 9 ARGs signature of patients with GC in TCGA and GEO. a, b The RS distribution, vital status of patients and heatmap of the 9 ARGs expression profiles between high risk group and low risk group in training or validation group. c, e Kaplan-Meier analysis of the prognostic model in TCGA or GEO datasets. $\mathbf{d}$, $\mathbf{f}$ Time-dependent ROC analysis showing the optimal AUC of the gene signature in the two cohorts

neovascularization will provide oxygen and energy to the tumor and further promote tumor growth. Angiogenic genes play an important role in tumor growth and prognosis.

With the wide application of high-throughput array, the combinatorial analysis of angiogenic genes involved in the carcinogenicity of GC has been realized [22]. In this study, we collected transcriptional expression data and corresponding clinical data from TCGA and GEO databases. Then the differential expression of ARGs between GC samples and nontumor samples were obtained. Finally, a prognostic model based on 9 prognosis-related ARGS (AGT, ANGPT1, SERPINE1, ANGPT2, PVT1, PROCR,
KIT, PLAUR and CAV1) was constructed, showing a good prognostic value in TCGA and GEO databases.

These differentially expressed ARGs are mainly concentrated in P13K-Akt, Rap1 and MAPK pathways and involved in the angiogenesis of GC. PI3K/AKT is an important intracellular signal transduction molecule, which participates in the regulation of cell proliferation, apoptosis and differentiation, and can regulate the expression of VEGF and hypoxia inducible factor (HIF-1) by activating kinases p70S6K1 and HDM2 [23]. VEGF is the main regulatory factor involved in tumor angiogenesis, while hypoxia can stimulate the secretion of angiogenic factors. Besides, PI3K-Akt pathway also plays an important role in hematopoiesis and 

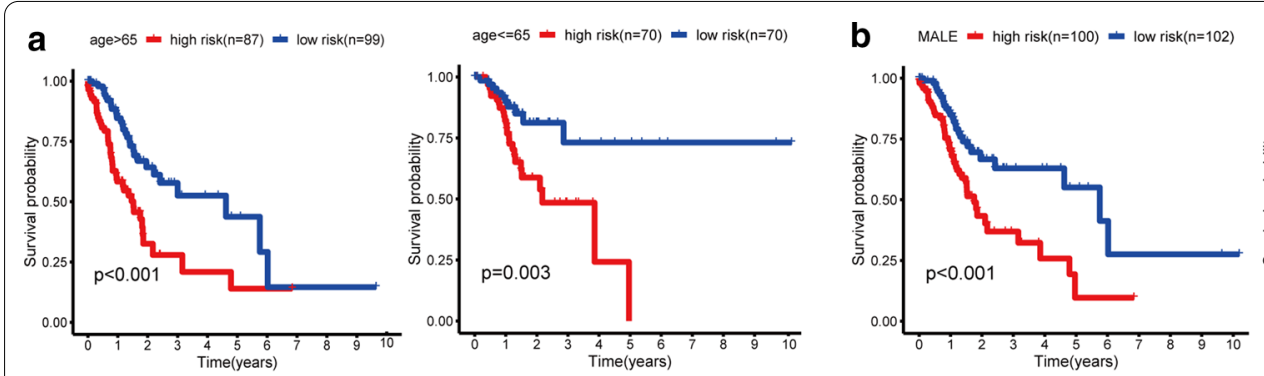

FEMALE + high risk(n=57) $\doteq$ low risk(n=67)
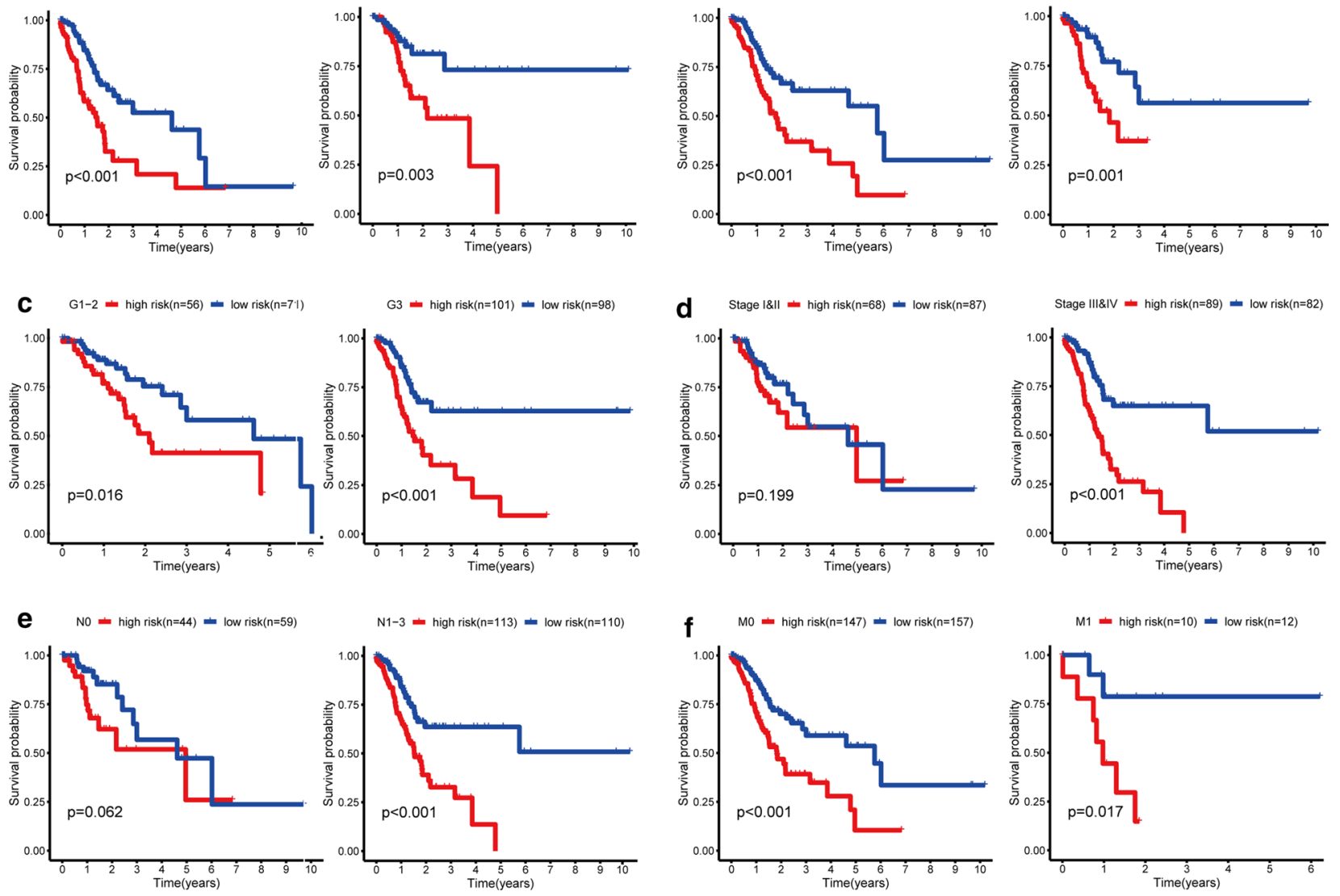

Fig. 6 Stratified analysis of the relationship between RS score and survival rate of patients with gastric cancer in TCGA cohorts. a Age $>65$ years and age $\leq 65$ years. b female sex and male sex. c G1-2 and G3. $\mathbf{d}$ Stage I\&II and stage IIIII. e NO stage and N1-3 stage. (f) M0 stage and M1 stage

angiogenesis mediated by K-ras signal pathway [24]. MAPK signal pathway is one of the important signal transduction systems in organisms, which plays an important role in cell survival, proliferation and angiogenesis [25]. A large number of studies have shown that RAP1 is activated in a variety of cancers, including leukemia and solid tumors [26, 27]. RAP1 plays a role in the invasion and metastasis of various tumor cells by regulating adhesion junctions and cytoskeleton remodeling.

Nine ARGs were used to establish the model equations for risk assessment. Among them, 4 candidate genes (ANGPT2, ANGPT1, PVT1, PROCR) were already reported to promote $\mathrm{GC}$ angiogenesis and tumorigenesis. ANGPT1 and ANGPT2 belong to the angiopoietin family, which play a central role in angiogenesis and are highly expressed in a variety of tumors such as GC, breast cancer and lung cancer [28-30]. Zhao et al. confirmed that PVT1 was upregulated and significantly associated with high-microvessel density and poor prognosis in GC [31]. Furthermore, overexpression of PVT1 in GC significantly increased the expression levels of angiogenesis-related transcription factors (STAT3, VEGFA, CTGF, ANGPT2) [32]. PROCR can promote tumor angiogenesis in vitro by activating ERK1/2 and AKT in GC cells, dependent on the activation of PAR1 [33].

It has already been proved that CAV1, SERPINE1 and AGT played important roles in the proliferation, migration and invasion of GC cells. What's more, their expressions in tumors were candidate prognostic biomarkers for GC patients. Data from Gene Expression Profiling Interactive Analysis (GEPIA) revealed similar results. Wang et al. found that $\mathrm{CAV}-1$ promoted drug resistance of GC cells through PI3K/Akt and MEK/ ERK signaling pathways [34]. Another study indicated that positive Cav-1 expression in GC patients was associated with poor prognosis after radical gastrectomy 

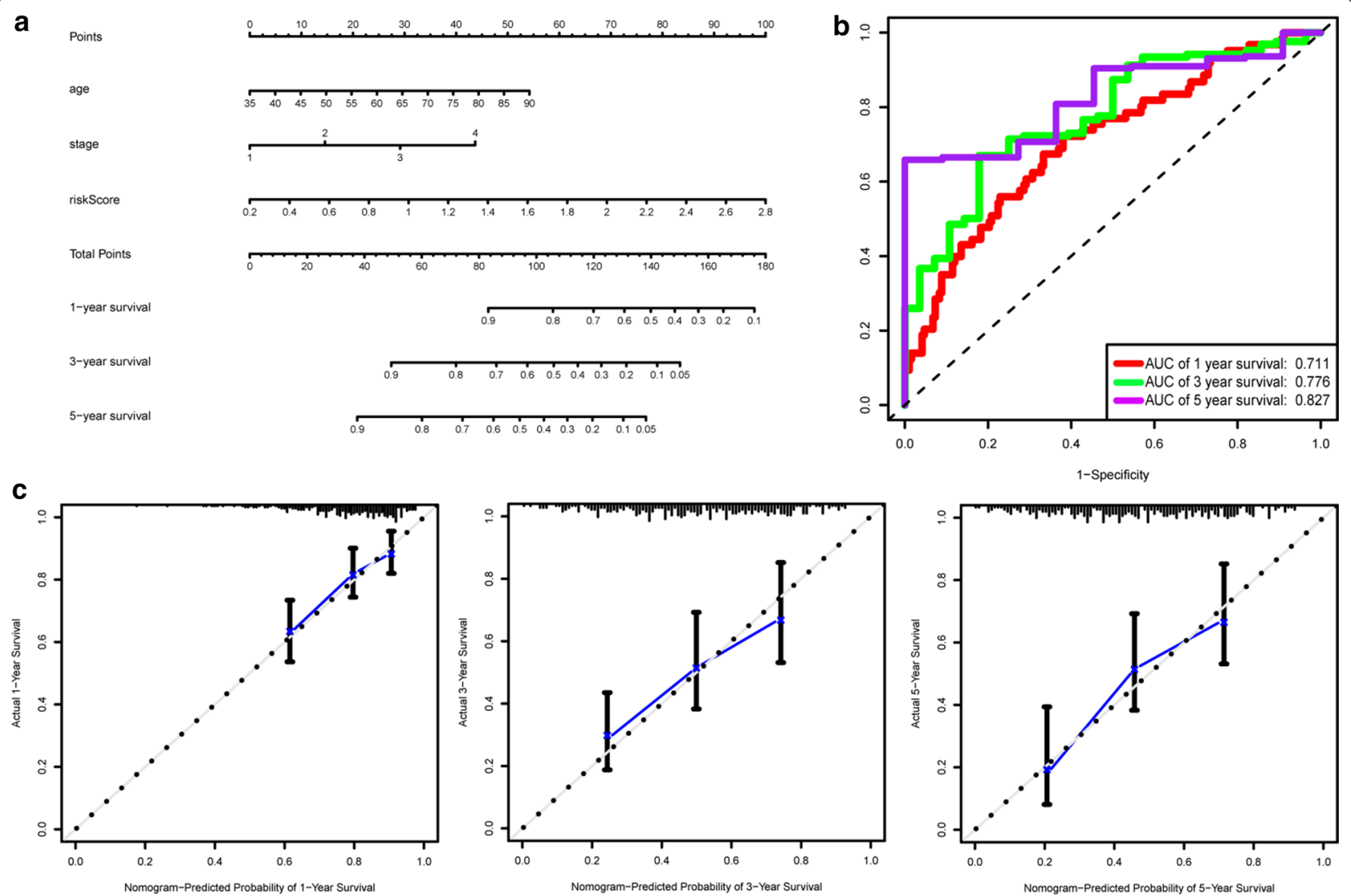

Fig. 7 Nomogram for predicting of 1-, 3- and 5-year overall survival (OS) based on the nine ARGs signature. a A nomogram based on the risk scores, clinical stage and age of GC patients. $\mathbf{b}$ ROC analysis of the nomogram for predicting the 1-, 3- and 5-year OS. c Calibration curves of nomogram for survival prediction at 1-, 3- and 5-year

$[35,36]$. Plasminogen activator urokinase receptor (PLAUR) can be used as predictors of aggressive phenotypes in preoperative biopsies, Helicobacter pylori infection, and intestinal metaplasia [37]. The mutation of KIT is an important mechanism in gastrointestinal stromal tumors (GIST), but its role in GC is still unclear [38]. Further research on the roles of these genes in GC is still required.

So far, most of the tumor-related genes identified in bioinformatics methods were analyzed separately, which cannot fully reflect the process of tumorigenesis, and the role in the diagnosis and prognosis prediction is still poor. In addition, most studies focused on a single cancer, rather than on one specific type of cancer or one specific process in carcinogenesis. It will be more valuable to identify a cluster of genes with prognosis function in one specific cancer-related process. We generated a multigene signature in this study to predict the prognosis of individual GC patients, focusing on the tumor angiogenesis sets. However, this study also has some shortcomings. First of all, we checked the data in the public database, so the study could be more valuable if further experiments in GC cells and animal models are performed on these genes. Secondly, the validation group in this study is based on GEO database. The conclusions could be more powerful after being verified in a separate cohort.

In conclusion, our study confirmed the relevant genes and pathways in the angiogenesis process of GC. They could work as potential biomarkers to predict the prognosis and diagnosis of GC and provide new perspectives in targeted therapy. 


\section{Supplementary Information}

The online version contains supplementary material available at https://doi. org/10.1186/s12876-021-01734-4.

Additional file 1: Supplementary Table I. Baseline clinical characteristics of patients with gastric cancer in this research. Supplementary Table $\mathbf{2}$ The prognostic related ARGS screened by univariate Cox regression analysis. Supplementary Fig. 1. Differentially expression and prognosis of VEGFA in gastric cancer. (a) Differentially expressed VEGFA between GC and normal gastric tissues from TCGA cohort. (b) Kaplan-Meier analysis of the prognosis of VEGFA in TCGA cohort. (c) Kaplan-Meier analysis of the prognosis of VEGFA in GEO cohort.

\section{Authors' contributions}

SZ, ZZZ and JWS conceived and designed the study. JYC, LMS and YFL Collected and analyzed the data from public datasets. SZ, JZ and ND wrote the manuscript. ZHL provided suggestions according to the revision requested. MX revised the manuscript. All authors read and approved the final manuscript.

\section{Funding}

This work was supported by the National Natural Science Foundation of China (82073229, 81702308 and 81802348), and the Natural Science Foundation of Zhejiang Province (LY18H160009 and LY16H160031).

\section{Availability of data and materials}

The datasets used and/or analyzed during the current study are available from TCGA repository: https://portal.gdc.cancer.gov/; GEO repository: https:// www.ncbi.nlm.nih.gov/geo/; the GeneCards website: https://www.genecards. org/; the STRING database: http://www.string-db.org/; and the KEGG pathway database: www.kegg.jp/kegg/kegg1.html.

\section{Declarations}

\section{Ethics approval and consent to participate}

None.

\section{Consent for publication}

A copy of the written consent is available for review by the Editor of this journal.

\section{Competing interests}

None of the authors has any conflict of interest to disclose.

\section{Author details}

${ }^{1}$ Department of Gastroenterology, The Second Affiliated Hospital of Zhejiang University School of Medicine, 88 Jiefang Road, Hangzhou 310009, Zhejiang, China. ${ }^{2}$ Institute of Gastroenterology, Zhejiang University, Hangzhou, China.

\section{Received: 9 October 2020 Accepted: 22 March 2021}

Published online: 01 April 2021

\section{References}

1. Bray F, Ferlay J, Soerjomataram I, et al. Global cancer statistics 2018: GLOBOCAN estimates of incidence and mortality worldwide for 36 cancers in 185 countries. CA Cancer J Clin. 2018;68(6):394-424.

2. Ilson $\mathrm{DH}$. Angiogenesis in gastric cancer: hitting the target? Lancet. 2014;383(9911):4-6.

3. Sisic L, Strowitzki MJ, Blank S, et al. Postoperative follow-up programs improve survival in curatively resected gastric and junctional cancer patients: a propensity score matched analysis. Gastric Cancer. 2018;21(3):552-68.

4. Sisic L, Blank S, Nienhuser $H$, et al. The postoperative part of perioperative chemotherapy fails to provide a survival benefit in completely resected esophagogastric adenocarcinoma. Surg Oncol. 2020;33:177-88.
5. Zhao $L$, Jiang $L, H e ~ L$, et al. Identification of a novel cell cycle-related gene signature predicting survival in patients with gastric cancer. J Cell Physiol. 2019:234(5):6350-60

6. Staton CA, Reed MW, Brown NJ. A critical analysis of current in vitro and in vivo angiogenesis assays. Int J Exp Pathol. 2009;90(3):195-221.

7. Rajabi M, Mousa SA. The role of angiogenesis in cancer treatment. Biomedicines. 2017:5(2):34.

8. Carmeliet P, Jain RK. Angiogenesis in cancer and other diseases. Nature. 2000;407(6801):249-57.

9. LiT, Kang G, Wang T, et al. Tumor angiogenesis and anti-angiogenic gene therapy for cancer. Oncol Lett. 2018;16(1):687-702.

10. Ramjiawan RR, Griffioen AW, Duda DG. Anti-angiogenesis for cancer revisited: is there a role for combinations with immunotherapy? Angiogenesis 2017:20(2):185-204.

11. Viallard C, Larrivee B. Tumor angiogenesis and vascular normalization: alternative therapeutic targets. Angiogenesis. 2017;20(4):409-26.

12. Nienhuser $H$, Schmidt T. Angiogenesis and anti-angiogenic therapy in gastric cancer. Int J Mol Sci. 2017;19(1):43.

13. Wilke H, Muro K, Van Cutsem E, et al. Ramucirumab plus paclitaxel versus placebo plus paclitaxel in patients with previously treated advanced gastric or gastro-oesophageal junction adenocarcinoma (RAINBOW): a double-blind, randomised phase 3 trial. Lancet Oncol. 2014;15(11):1224-35.

14. Pang L, Wang J, Fan Y, et al. Correlations of TNM staging and lymph node metastasis of gastric cancer with MRI features and VEGF expression. Cancer Biomark. 2018;23(1):53-9.

15. Tibshirani R. Regression shrinkage and selection via the Lasso. J R Stat Soc B Met. 1996;58(1):267-88.

16. Sauerbrei W, Royston $\mathrm{P}$, Binder $\mathrm{H}$. Selection of important variables and determination of functional form for continuous predictors in multivariable model building. Stat Med. 2007;26(30):5512-28.

17. Kidd AC, McGettrick M, Tsim S, et al. Survival prediction in mesothelioma using a scalable Lasso regression model: instructions for use and initial performance using clinical predictors. BMJ Open Respir Res. 2018;5(1):e000240.

18. Song $Z, W u Y$, Yang J, et al. Progress in the treatment of advanced gastric cancer. Tumour Biol. 2017;39(7):1010428317714626.

19. Zhang D, Zhou S, Liu B. Identification and validation of an individualized EMT-related prognostic risk score formula in gastric adenocarcinoma patients. Biomed Res Int. 2020;2020:7082408.

20. Carmeliet P. Angiogenesis in life, disease and medicine. Nature. 2005;438(7070):932-6.

21. Carmeliet $P$, Jain RK. Molecular mechanisms and clinical applications of angiogenesis. Nature. 2011;473(7347):298-307.

22. Sammarco G, Varricchi G, Ferraro V, et al. Mast cells, angiogenesis and lymphangiogenesis in human gastric cancer. Int J Mol Sci. 2019;20(9):2106.

23. Skinner HD, Zheng JZ, Fang J, et al. Vascular endothelial growth factor transcriptional activation is mediated by hypoxia-inducible factor 1alpha, HDM2, and p70S6K1 in response to phosphatidylinositol 3-kinase/AKT signaling. J Biol Chem. 2004;279(44):45643-51.

24. Liu L, Zhu S, Gong Z, et al. K-ras/PI3K-Akt signaling is essential for zebrafish hematopoiesis and angiogenesis. PLoS ONE. 2008;3(8):e2850.

25. Teng F, Xu Z, Chen J, et al. DUSP1 induces apatinib resistance by activating the MAPK pathway in gastric cancer. Oncol Rep. 2018;40(3):1203-22.

26. Minato N. Rap G protein signal in normal and disordered lymphohematopoiesis. Exp Cell Res. 2013;319(15):2323-8.

27. Shah S, Brock EJ, Ji K, et al. Ras and Rap1: A tale of two GTPases. Semin Cancer Biol. 2019:54:29-39.

28. Chen Z, Zhu S, Hong J, et al. Gastric tumour-derived ANGPT2 regulation by DARPP-32 promotes angiogenesis. Gut. 2016;65(6):925-34.

29. Lin CY, Cho CF, Bai ST, et al. ADAM9 promotes lung cancer progression through vascular remodeling by VEGFA, ANGPT2, and PLAT. Sci Rep. 2017;7(1):15108.

30. Makhoul I, Todorova VK, Siegel ER, et al. Germline Genetic Variants in TEK, ANGPT1, ANGPT2, MMP9, FGF2 and VEGFA Are Associated with Pathologic Complete Response to Bevacizumab in Breast Cancer Patients. PLoS ONE. 2017;12(1):e0168550.

31. Zhao J, Du P, Cui P, et al. LncRNA PVT1 promotes angiogenesis via activating the STAT3/VEGFA axis in gastric cancer. Oncogene. 2018;37(30):4094-109. 
32. Zheng J, Hu L, Cheng J, et al. IncRNA PVT1 promotes the angiogenesis of vascular endothelial cell by targeting miR26b to activate CTGF/ANGPT2. Int J Mol Med. 2018;42(1):489-96.

33. Wang $Q$, Tang $Y$, Wang T, et al. EPCR promotes MGC803 human gastric cancer cell tumor angiogenesis in vitro through activating ERK1/2 and AKT in a PAR1-dependent manner. Oncol Lett. 2018;16(2):1565-70.

34. Wang K, Zhu X, Mei D, et al. Caveolin-1 contributes to anoikis resistance in human gastric cancer SGC-7901 cells via regulating Src-dependent EGFRITGB1 signaling. J Biochem Mol Toxicol. 2018;32(10):e22202.

35. Seker M, Aydin D, Bilici A, et al. Correlation of Caveolin-1 expression with prognosis in patients with gastric cancer after gastrectomy. Oncol Res Treat. 2017:40(4):185-90.

36. Ye Y, Miao SH, Lu RZ, et al. Prognostic value of caveolin-1 expression in gastric cancer: a meta-analysis. Asian Pac J Cancer Prev. 2014;15(19):8367-70.
37. Beyer BC, Heiss MM, Simon EH, et al. Urokinase system expression in gastric carcinoma: prognostic impact in an independent patient series and first evidence of predictive value in preoperative biopsy and intestinal metaplasia specimens. Cancer. 2006;106(5):1026-35.

38. Theiss L, Contreras CM. Gastrointestinal stromal tumors of the stomach and esophagus. Surg Clin North Am. 2019;99(3):543-53.

\section{Publisher's Note}

Springer Nature remains neutral with regard to jurisdictional claims in published maps and institutional affiliations.
Ready to submit your research? Choose BMC and benefit from:

- fast, convenient online submission

- thorough peer review by experienced researchers in your field

- rapid publication on acceptance

- support for research data, including large and complex data types

- gold Open Access which fosters wider collaboration and increased citations

- maximum visibility for your research: over $100 \mathrm{M}$ website views per year

At BMC, research is always in progress.

Learn more biomedcentral.com/submissions 